\title{
Inclusión social vs responsabilidad social impacto que proporciona en procesos de paz
}

Social inclusion vs. social responsibility impact it provides in peace processes

Paula Andrea León Silva

Estudiante de administración de empresas, Universitaria Agustiniana - Uniagustiniana.

Correo electrónico: paula.leons@uniagustiniana.edu.co 


\section{Resumen}

En el siguiente artículo se rescatará la importancia y el concepto de inclusión, relacionándolo con el proyecto denominado Centro de Capacitación para Personas Vulnerables enfocado en Desmovilizados (CCPVD), identificando la responsabilidad social de las empresas de todos los sectores económicos frente a la inclusión social, en este caso con relación a los diferentes actores involucrados en el actual proceso de paz firmado por el gobierno colombiano con el grupo insurgente de las Farc. Tomando como punto de partida, los procesos de paz firmados por otros países como: Honduras, Salvador, Perú y México; donde se resalta, que para cumplir el objetivo, de proceso de paz, se deben mantener de manera constante, diálogos para pactar y establecer diferentes aspectos que influyan en el momento de la firma, haciendo énfasis en cómo se ha manifestado la responsabilidad social empresarial (RSE), en la incorporación de la población desmovilizada; siendo está excluida y discriminada por sus antecedentes judiciales, del mismo modo ha presentado de manera constante dificultades en su incorporación al momento de integrarse a un nuevo ambiente laborales. Por ello se quiere identificar las oportunidades, competencias y estrategias que han implementado diferentes gobiernos luego de la firma de procesos de paz, participación del sector productivo y educativo en el desarrollo de estrategias para afrontar el reto de incorporar a las personas en condición de reinsertadas a la vida civil.

Palabras clave: tratados de paz, responsabilidad social, desmovilizados, inclusión social, reinsertados, emprendimiento.

\section{Abstract}

In the following article you will rescue the importance and the concept of inclusion, relating it to the project entitled training Centre for Vulnerable People Focused on Demobilized (CVPFD), identifying the social responsibility of enterprises in all economic sectors against the social inclusion, in this case with relation to the different actors involved in the current process of peace signed by the Colombian government with the group insurgent of the Revolutionary Armed Forces of Colombia (RAFC). Taking as a starting point, the processes of peace signed by other countries such as: Honduras, Salvador, Peru and Mexico; where is highlighted, that to meet the objective of the peace processes, should be so constant dialogues to negotiate and establish different aspects influencing the time of signing, emphasizing how has been the corporate social responsibility (CSR) in the incorporation of demobilized combatants; being excluded and discriminated against because of its judicial background, in the same way it has presented a constant difficulty in its incorporation when integrating to a new work environment. Why you want to identify the opportunities, skills and strategies that have been implemented by different Governments after the signing of the processes of peace, participation of the productive and educational sector in the development of strategies to face the challenge of incorporating people in a condition of reinserted into life civil.

Keywords: treaties of peace, social responsibility, demobilized, social inclusion, reinserted, entrepreneurship.

\section{Introducción}

Durante el transcurso del tiempo, algunos países han tenido que afrontar, con la creación de grupos de izquierda, una forma de violencia que sin lugar a duda ha afectado de manera directa la seguridad e integridad de la población, por esta razón el gobierno ha implementado técnicas que faciliten y ayuden a resolver y enfrentar esta realidad, estableciendo e identificando estrategias y oportunidades, con el propósito de llegar a un acuerdo que 
beneficie al país en general. El objetivo de este artículo es hallar posibles obstáculos que se pueden presentar al momento de firmar el proceso de paz entre Colombia y las Farc, tomándolo como referencia.

Teniendo en cuenta lo anterior, Colombia ha tenido que sufrir por más de 70 años situaciones de violencia generada por las acciones de los diferentes grupos conformados al margen de la ley; donde unos actores se han visto más perjudicados que otros, por ejemplo, padres que sufren la pérdida de hijos que prestan sus servicios como soldados o policías, personas secuestradas, campesinos víctimas del conflicto, entre otros; dejando familias con duelos muy grandes o madres y familiares que aún no saben cuál fue el destino de sus hijos que desaparecieron prestando su servicio militar; otro es el dolor de las personas que deben afrontar una realidad frente al reclutamiento forzoso de seres queridos, que en ocasiones no se vuelven a ver; y demás acciones dolorosas que ha dejado el tema del conflicto de las guerrillas y el paramilitarismo en el país.

La finalidad de este artículo es hallar la relación entre dos términos: inclusión social, siendo esta la incorporación de la población más vulnerable (Araoz, 2010) y responsabilidad empresarial, el compromiso general para resarcir el daño que se puede ocasionar con la actividad económica que se esté realizando (Duque, Cardona \& Rendón, 2013). Donde se tiene como punto focal el cómo contribuir para disminuir la desigualdad y violencia que ha tenido que afrontar el país, y del mismo modo sensibilizar a la población en general a diseñar estrategias de fortalecimiento. Identificar cómo las empresas pueden apoyar el proceso por medio de su gestión en cuanto a Responsabilidad Social Empresarial.

Partiendo de la problemática expuesta anteriormente y de las acciones que han llevado a cabo diferentes gobiernos, todos los ciudadanos, incluyendo los denominados millennials, tienen un compromiso frente al proceso de paz siendo conscientes de las problemáticas que afectan la ar- monía, comunicación y participación del país, por tanto, se puede proporcionar posibles soluciones interviniendo y realizando estrategias para el mejoramiento y fortalecimiento de la calidad de vida y del mismo modo logrando la disminución de grupos de violencia.

\section{Antecedentes}

\section{Guatemala: Unidad Revolucionaria Nacional Guatemalteca (URNG)}

En 1982 se formó la Unidad Revolucionaria Nacional Guatemalteca (URNG), se formó inicialmente en la clandestinidad, en el contexto de la guerra civil, como una organización político-militar, coordinadora de las cuatro organizaciones guerrilleras, la cuales eran el Ejército Guerrillero de los Pobres (EGP); las Fuerzas Armadas Rebeldes (FAR); la Organización del Pueblo en Armas (ORPA), y el Partido Guatemalteco del Trabajo (PGT), que desde principios de la década de los años 70 se habían alzado en armas contra el Estado, con el objetivo de tomar el control del poder y llevar a cabo una revolución política y social en Guatemala. Este mismo año se presentó un golpe de Estado donde Efraín Ríos Montt asume el poder, en lucha contra la población civil, bajo pretexto de quitarle bases a la URNG, lo que da como resultado un sin número de desapariciones y asesinatos.

Después de 36 años de conflicto armado interno que vivió la población guatemalteca, se presentaron negociaciones que fueron establecidas entre 1991 y 1996, la URNG, tres gobiernos y el ejército de Guatemala firmaron una serie de acuerdos de varios tipos (de procedimiento, operativos y sustantivos), el objetivo de llegar a acuerdos que implicaran transformaciones estructurales en el país y no solamente el fin del conflicto. Finalmente, el 29 de diciembre de 1996 se llevó a cabo en Guatemala la firma de la paz. Uno de los principales resultados fue la reforma Constitucional para organizar las funciones del ejército, como también 
la desmovilización de las fuerzas guerrilleras y la reinserción de la dirigencia y militancia en la vida política y ciudadana. En el contexto de lo establecido en tales acuerdos, URNG se convirtió el 18 de diciembre de 1998 en un partido político, lo cual la habilitó para participar en el proceso electoral de 1999.

La fase de implementación del proceso de paz fue algo difícil para este país, pero es importante reconocer que a pesar de los obstáculos que de alguna u otra forma se presentaron, se pudo observar beneficios y avances importantes; los tratados de paz se ha convertido en una etapa histórica para la comunidad indígena siendo esta la más perjudicada por los antecedentes y lucha entre el gobierno y grupos armados, con lo que aumentó la participación de la población indígena en sus comunidades, se empezaron a manifestar en defensa de los derechos de la mujer, aunque con muchos obstáculos y dificultades, pero, se han reposicionado para defender sus derechos y han logrado avanzar en la agenda nacional de sus necesidades, especialmente en el tema de violencia, donde no solo se ha legislado sino se han creado tribunales especializados. La participación ciudadana aumentó considerablemente, ya que el principal objetivo era garantizar y proteger el respeto a los derechos humanos, por tanto, es evidente la pérdida del miedo al expresarse, a manifestarse, a protestar, asuntos que eran imposibles antes de la firma de la paz (Proceso de paz en: un estudio de caso sobre la negociación y el proceso de diálogo nacional en Guatemala, 2016).

Experiencias útiles al caso de Colombia. Colombia y Guatemala presentan numerosas diferencias que podrían afectar la garantía de la estrategia del proceso de paz. Estas incluyen aspectos relevantes como el tamaño del país, el número de habitantes, la complejidad y el número de actores involucrados, recursos nacionales, el origen del conflicto, y por supuesto, el narcotráfico como fuente de recursos para la guerrilla, sobre lo que es importante resaltar que Colombia ha recibido grandes ingresos provenientes del narcotráfico, dinero que reciben unos pocos, pero que genera un enorme daño no sólo a la población, sino a la imagen del país a nivel internacional, lo cual incluso influye en la calificación que se le da al país, por parte de las entidades a nivel internacional.

Pero asimismo, existen una serie de similitudes, que podrían ser un apoyo para influir en la firma de paz en Colombia; como el agotamiento de la población por la creación y enfrentamiento entre grupos de izquierda y el gobierno, así mismo tuvieron que evidenciar innumerables problemas a nivel interno, como también muertes y desastres para la población más vulnerables, siendo esta, la que más se ve expuesta a esta situación y de alguna forma obligada a colaborar con alguna de las partes en conflicto.

\section{Sierra Leona: Frente Revolucionario} Unido (FRU)

En 1991 Sierra Leona tuvo que enfrentarse y vivir de manera progresiva desastres, masacres, e intervenciones en su diario vivir, ya que se creó el Frente Revolucionario Unido (FRU) para aponerse y destituir al gobierno de ese entonces, a comienzos de estos duelos el Grupo de Observadores Militares (ECOMOG) de la Comunidad Económica de los Estados de África Occidental (CEDEAO), tratan de apoyar y defender al gobierno.

Con lo anterior ,el gobierno no tuvo otra opción que darse de baja, pero, a pesar del suceso FRU siguió con las desastrosas acciones que había realizado desde un comienzo, por lo tanto en 1995 la Naciones Unidas designaron a Berhanu Dinka, el que se encargaría de iniciar diálogos con FRU, recibiendo apoyo de la Organización de la Unidad Africana (OUA) y de la CEDEAO, identificado sus ideales y del mismo modo buscar alternativas que los ayudara a la negociación con el fin de establecer el régimen civil del país.

En 1996 se presentan nuevas elecciones, se pensaba que tal vez el grupo armado FRU iba acceder por el cambio gubernamental pero no fue así el conflicto continuo, pero esto no fue un impedi- 
mento para seguir los diálogos y negociaciones teniendo como nombre acuerdo de Abidján, este fracasando por que se presentó un nuevo golpe de estado militar en 1997, por tanto, el ejército se unió con FRU y realizaron una junta de gobierno.

En 1997 CEDEAO ideó un plan con pautas y algunos beneficios que tendrían este grupo (FRU), si en algún caso estarían de acuerdo en no seguir exponiéndose a actividades delictivas. Esta junta mantuvo conversaciones hasta cumplir con todas las expectativas de cada miembro y del mismo modo, buscando soluciones a cada aspecto negativo que se evidenciaba en el país; para ponerlo en práctica debía ser aprobada por el Consejo de Seguridad de la ONU y recibir la asistencia de observadores militares de las Naciones Unidas.

Tras consecutivos ataques el representante, Sr. Okelo, en consulta con Estados del oeste africano, se inició en 1999 diálogos con FRU, en donde las alternativas de cambio fueron expuestas tanto por el gobierno como el grupo FRU y el 7 de julio de ese mismo año fue firmado el tratado, con el fin de cesar las hostilidades y formar un gobierno de unidad nacional. Uno de los aspectos más importantes que se tuvieron en cuenta fue la intervención de comunidades internacionales.

"No puede haber una auténtica reconciliación y con ella, una paz duradera si no se establece la verdad sobre los graves abusos contra los derechos humanos que se han cometido en Sierra Leona y sus autores no rinden cuentas de sus actos", por ende, es importante que cada miembro que participe en actos inmorales y que afecte de alguna u otra forma la seguridad de la sociedad debe pagar por cada acto delictivo (Fisas, 2010).

\section{El Salvador: Farabundo Martí de Liberación Nacional (FMLN)}

En los años 80, en El Salvador aparecen diversos grupos armados, debido a las reformas fundadas por la Junta Revolucionaria del gobierno, esto dan- do como resultado el conflicto entre estos grupos con el fin de solucionar aspectos determinantes del conflicto social salvadoreño.

En 1891 se evidenció un aumento en las acciones militares por parte del ejército y el Farabundo Martí de liberación nacional (FMLN), por lo tanto en 1984 se ve la necesidad de iniciar conversaciones con apoyo de la Iglesia, donde se propusieron algunas alternativas de solución, centrándose en aspectos específicos, donde se estipulan los logros y compromisos de cumplimiento obligatorio, pero en 1987 se suspende el acuerdo de paz dirigido por el presidente Duarte por cumplimiento de fase gubernamental.

Se vuelven a establecer los diálogos de paz cuando sube a la presidencia el licenciado Alfredo Cristiani, el cual tenía relación con militares y la derecha salvadoreña, en 1989 se consolida el apoyo a nivel internacional y del mismo modo una mayor perspectiva tras la perestroika y la caída del muro de Berlín. Al iniciar nuevamente las conversaciones, el presidente había establecido elementos con su respectiva duración, por lo cual FMLN no estuvo de acuerdo empezando así a realizar atentados $y$ actividades delincuenciales ya que no se encontraba el punto de equilibrio donde las dos partes cumplieran con sus expectativas, después de estos medios de violencia el dialogo se culminó.

En 1990 se iniciaron nuevamente proceso de reconciliación, como el llamado acuerdo de Ginebra, (Fisas, 2010), uno de los puntos que se tuvieron en cuenta fueron la reforma a la constitución, esto creando una crisis en la negociación. Se realizaron avances significativos en 1991 donde se acordaron puntos sociales, económicos y políticos y se aborda el tema del cese al fuego, bajo la accesoria de la Comisión Económica para América Latina (CEPAL).

Después de 25 años fue firmado el 16 de enero del 1992 el tratado; para su regulación, siguiendo los términos pactados, fueron creadas entidades de vigilancia para hacer cumplir cada propuesta y del mismo modo teniendo como prioridad los derechos humanos. El reintegro no fue nada fácil ya 
que se manifestaron destrucciones de las tierras de la población desmovilizada, esto no solo afectó, a esta población, también a los campesinos ya que disminuyeron las tierras cultivables.

Este acuerdo dio como resultado grandes beneficios para todas las entidades que intervienen, como lo es la iglesia, el ejército y los partidos políticos, teniendo en cuenta que para hacer valer sus derechos deben responder con sus deberes, demostrándolo de la manera más eficaz y responsable, buscando alternativas de apoyo para lograr su objetivo. Hubo importantes cambios, entre ellos, la privatización de la banca, la energía, las telecomunicaciones y las pensiones, entre otras, así como la dolarización de la economía y un esfuerzo por disminuir el tamaño de un Estado históricamente débil. Aún, así el país siguió siendo violento ya que El Salvador tiene cuatro fracturas: la política, la economía, y social e histórica, lo cual tiene un grado de complejidad elevado para poder efectuar un cambio; el cual puede ser posible si todas las fracturas tienen presente el beneficio comunitario (Veléz, 2017).

El conflicto en el Salvador, motivó el desarrollo de la película Las voces inocentes (Mandoki, 2005), la cual fue creada con el fin de mostrar el conflicto de la guerra civil que enfrentó El Salvador, el cual comenzó con un conflicto agrario y terminó convirtiéndose en un brutal enfrentamiento entre el ejército salvadoreño y los campesinos que se organizaron con el movimiento guerrillero el Frente Farabundo Martí para la Liberación Nacional (FMLN). Es una película que evidencia y muestra la realidad que vivió El Salvador, un filme que en muchos temas académicos invita a la reflexión para llegar a comprender el problema que para muchos es sólo del gobierno.

\section{México: Movimiento Zapatista}

El movimiento Zapatista se crea en 1994, principalmente porque la cultura indígena era apartada y poco reconocida en las decisiones del gobierno, tenían una ideología marxista con el objetivo de proporcionarle beneficios directos a la cultura indígena, ya que el TLC se convierte en una amenaza debido a que los pequeños productores serían arrastrados por el ingreso de nuevos mercados, por la falta de conocimiento y comprensión de la ideología marxista vieron la necesidad de cambiar esta situación. La percepción de la cultura indígena ayudó a que el movimiento Zapatista interviniera y fuera mucho más fácil su organización con el fin de defender sus derechos (Alonso, 2003).

El EZLN se pronunció después de intentar defender de forma apacible los derechos de la cultura indígena "el camino de fuego se abrió ante la imposibilidad de luchar pacíficamente por los derechos elementales del ser humano. Siendo violado de manera consecutiva la libertad y de participación en forma democrática" (Auger, 2013), por tanto, se identifica la falta de compromiso del gobierno al momento de tomar decisiones que no perjudicaran la integridad y estabilidad de vida de esta población vulnerable (indígenas), por lo que vieron la necesidad de demostrar el rango de inconformidad realizando un golpe en la estabilidad y del mimo modo en la forma de vida de cada integrante de este país.

Como respuesta al suceso, el gobierno realiza alternativas de oposición de manera inmediata con más guerra, afirmando que no existía presencia indígena en la agenda nacional, examinando aspectos que dieran un impacto en el comportamiento de este movimiento, desprestigiándose y dando acusaciones invalidas. El EZLN después de las acusaciones que realizó el gobierno de este país decide cambiar las armas por las palabras, donde las armas serán utilizadas exclusivamente si se presentaba algún enfrentamiento hacia la población indígena, así dando origen a la unión de redes comunitarias con base a las clases sociales y étnicas.

El proceso de paz comienza en 1996 con la asociación de diferentes grupos indígenas como el movimiento Zapatista, grupos de violencia 
formados con la finalidad de tener en cuenta a la población indígena, también disminuir la desigualdad y buscar el bienestar de la población más pobre. Estos diálogos debían garantizar los derechos obligatorios de la población indígena por ende se crea una ley especialmente con esta finalidad, la que no fue aprobada por el Congresos, dando como consecuencia la finalización de los diálogos y ocasionando lo creación en el 2003 de facto, siendo esta una estructura de autogobierno (Auger, 2013).

\section{Perú: Sendero Luminoso}

El Sendero Luminoso (PCP), fue un partido comunista creado en 1980, consecuencia de un prolongado enfrentamiento vanguardista y violento, con raíces en el marxismo-leninismo. Fue creado por el Frente Estudiantil Revolucionado (FER) contra el Estado y la población peruana, siendo el conflicto más fuerte que ha tenido que enfrentar este país, ya que esa agrupación realizó delitos y crímenes de lesa humanidad, siendo liderada por Abimael Guzmán, fue un factor decisivo en la crisis de la democracia peruana en 1992, ya que entidades que intervienen en las decisiones del gobierno presentaban dificultades a la hora de responder y enfrentarse a este grupo, del mismo modo los agentes y el Estado a causa de los enfrentamientos producían constantemente violaciones de manera directa a los derechos humanos y así mismo a un gran deterioro en las instituciones democráticas. Por ende, el antiguo tronco del Partido Comunista nació, de la Bandera Roja y Patria Roja, además de otros grupos maoístas que fueron Vanguardia Revolucionaria y el Movimiento de Izquierda Revolucionaria, menos dogmáticos que los anteriores, pero todos promotores de la vía armada.

Después de la captura del líder Abimael Guzmán y de la derrota de la guerrilla maoísta, se refleja la importancia de iniciar diálogos de paz, ya que para el grupo armado PCP, la prioridad era salvar la vida de su líder ante la derrota que se presentó con los militares del país y del mismo modo por la población campesina, por ende el 12 de septiembre de 1992 se inician los diálogos, teniendo en cuenta que Sendero Luminoso tenía gran relevancia en la política y las decisiones del gobierno (Padró, 2015).

Al firmarse este proceso se desataron opiniones de lado a lado, unos solicitando justicia, pero tanto en el caso de Colombia como de Perú, la persistencia del conflicto involucra una dificultad existente ante la minería ilegal, el narcotráfico, el crimen organizado y la trata de personas. Sin la voluntad de paz del Estado y de los grupos armados -que pueden ser catalogados desde insurgencias a grupos terroristas- la población civil acorralada entre ambos bandos continuará siendo el botín que amortiza el daño colateral de un conflicto redituable.

\section{Las Farc, proceso de paz con Colombia}

Partido político con orientación marxista-leninista, creado en 1946 que tenía como objetivo la implementación de un Estado comunista, con un gremio político, que planteaba defender los intereses de todos los sectores sociales, luchar contra la corrupción y fomentar las artes y la cultura, deja de ser partido político en 1964 y se convierte en un grupo terrorista, ya que existía de manera consecutiva la infracción de los derechos de los campesinos por parte del Estado (Pataquiva, 2009).

La lucha contra este grupo se empieza a manifestar de manera consecutiva durante el gobierno de Álvaro Uribe (2002), ya que empiezan a presentarse acciones directas de enfrentamientos entre este grupo armado y el gobierno, lo que obligó a las Farc, a retroceder. Durante el tiempo gubernamental de Uribe se presentan de forma paulatina enfrentamientos, muertes y asesinatos de personas inocentes y uno de los principales sucesos fue la masacre de Bojayá que tuvo aproximadamente 119 muertos, así como la masacre 
en el club El Nogal de Bogotá, en que se presentaron 36 muertos, siendo estos algunos de los sucesos, frente a lo cual el ejército asesinó a Iván Ríos, Raúl Reyes y Manuel Marulanda 'Tirofijo', el cofundador y cabecilla del grupo terrorista. Estas catástrofes y conflictos duraron 8 años, finalizando al terminar, el tiempo gubernamental de Álvaro Uribe (Pataquiva, 2009).

Cuando Juan Manuel Santos llega al gobierno, se empiezan a visualizar grandes cambios positivos, como el proceso de paz entre Colombia y las Farc, donde los puntos de discusión consistían en políticas de desarrollo agrario; participación política; fin del conflicto; solución al problema de drogas ilícitas; víctimas, y, por último el mecanismo de refrendación de los acuerdos, todo lo cual apuntaba a la dejación de armas por la Farc, con su ingreso y el reintegro a la vida civil, así como planes que concretan la suspensión de procesos judiciales a los subversivos; también aspectos específicos de cambio social, y que de alguna $\mathrm{u}$ otra forma iban a proporcionar un impacto directo en la calidad de la vida de la población. (Martín, 2017).

\begin{tabular}{|l|l|}
\hline Aspectos & Monto \\
\hline Reforma Rural Integral & 110,9 \\
\hline Participación política & 4,3 \\
\hline Fin del conflicto & 1,9 \\
\hline Drogas ilícitas & 8,3 \\
\hline Víctimas & 4,3 \\
\hline Total & $\mathbf{1 2 9 , 7}$ \\
\hline
\end{tabular}

Tabla 1. Costos totales de la implementación de acuerdo de paz (billones de pesos).

Fuente. Fedesarrollo, 2018.

Una de los temas expuestos por el presidente Juan Manuel Santos, fue la Reforma Rural, donde su objetivo consistía en realizar una inversión de 110,9 billones de pesos, teniendo como finalidad transformar de manera estructural el campo, para así darle un mayor impulso a la actividad económica primaria, la agricultura, esto apuntando al desarrollo del país; también se toma el tema de víctimas con un aporte de 4,3 billones de pesos que consistía en incluir a la población cam- pesina, la integración de las regiones, seguridad alimentaria y ofrecer alternativas de educación; para esto era importante el apoyo y conformismo de las Farc ya que la población se sentiría más segura si contaran con su ayuda.

La participación política, se toma cuando el gobierno le da la posibilidad al comandante Rodrigo Londoño de las Farc, alias Timochenko, de lanzarse a la presidencia, esto como un apoyo de reintegro, recibiendo un ingreso de 4,3 billones de pesos para su campaña política. Del mismo modo también se toma el fin de conflicto y de las drogas ilícitas con una contribución 10,2 billones de pesos con el objetivo de darle bienestar y una mejor calidad de vida a cada desmovilizado (Fedesarrollo, 2018). Este acuerdo busca mejorar la contribución de todos los colombianos en la política, los asuntos públicos y la construcción de la paz. Busca el incremento de la democracia como camino para gestionar los conflictos de manera tranquila y el rompimiento definitivo del vínculo entre política y armas, así como abrir la puerta para que en Colombia nos integremos a una cultura de reconciliación, convivencia, tolerancia y no estigmatización.

\section{¿Por qué hablamos de inclusión en educación?}

América Latina se caracteriza por tener sociedades muy desintegradas y fragmentadas, debido a la persistencia de la pobreza y a la gran desigualdad en la distribución de los ingresos, lo cual genera altos índices de exclusión. Como señala Reimers, 2002, una de las tendencias más fuertes de la nueva economía, es el aumento de las desigualdades, la segmentación espacial y la fragmentación cultura.

De este modo se puede decir que con el transcurrir del tiempo se iniciaron una serie de reformas educativas, orientadas a lograr el acceso a la educación básica y al mejoramiento de su calidad y equidad, sin embargo todavía persisten importantes desigualdades educativas a pesar del 
mejoramiento. También hay una discrepancia en función de la economía, y temas como la pobreza están asociados a otros tipos de distinción, por ejemplo, las personas que viven en zona rurales o viven en pueblos originarios; lo cual sitúa a un buen porcentaje de la población en una posición de gran vulnerabilidad (Blanco, 2006).

Las barreras al aprendizaje y la participación aparecen en la interacción entre el alumno y los distintos contextos: las personas, políticas, instituciones, culturas y las circunstancias sociales y económicas que afectan sus vidas; esto apuntando a la transformación y desarrollo de competencias, habilidades y solución de problemas. Para esto es importante la re-conceptualización de objetivos y metas que apunten al incentivo consecutivo de la investigación, por medio de los métodos de estudio esenciales para que cada persona apunte a la asimilación y entendimiento de temas de una manera más sencilla, dando libertad y confianza, con el objetivo de disminuir la discriminación y se dejen de presentar situaciones que fomenten el aislamiento causado por ser diferente (García, Aquino, Izquierdo \& Santiago, 2015).

\section{Responsabilidad social empresarial}

La responsabilidad social en una empresa consiste en, de forma voluntaria, integrar dentro de las operaciones comerciales y las relaciones con sus grupos de interés, los problemas sociales y medioambientales, afirmando, que una empresa no es socialmente responsable únicamente cuando cumple con sus obligaciones jurídicas, sino que debe ir más allá, pues debe aportar más en capital humano, en el entorno y en las relaciones con los actores sociales que intervienen en estos procesos (Comisión Europea, 2001).

De este modo la responsabilidad social empresarial, más que ser un compromiso es ayudar a personas vulnerables, brindándoles apoyo y oportunidades laborales y de reintegración con la sociedad civil, siendo importante capacitar a cada una de ellas, con el fin de poder tener una calidad de vida y ofrecer alternativas que apunten al crecimiento y solución de problemas sociales, por esta razón es primordial e indispensable que las empresas tomen conciencia y realicen alternativas de solución en aspectos económicos, sociales, y ambientales.

Para poder darle solución a este problema es importante tener en cuenta el ICBF, siendo un organismo que trabaja en conjunto con la organización internacional para las migraciones, con el objetivo de identificar el segmento o población con más vulnerabilidad. Con esto se ha evidenciado que, los más afectados son menores que están entre los 13 y 18 años. (Palacio, 2016). Por ende, las principales afectaciones psicosociales que presentan los niños desvinculados de los grupos criminales, se destacan alteraciones del pensamiento, retraimiento, ansiedad y depresión, problemas de conducta, problemas sociales, problemas de atención y de concentración. Por otro lado, en aquellos que fueron desplazados por la violencia, se encontraron problemas de salud mental asociados con retraimiento, ansiedad, problemas sociales y traumas.

Según datos del ICBF, aproximadamente 20.200 menores fueron desplazados de sus territorios por culpa de la violencia armada. Los departamentos que históricamente han presentado mayores índices por este fenómeno social son: Antioquia con 2.062 niños, Bolívar con 1.911; Tolima con 1.819; Caquetá con 1.502; Nariño con 1.279; Cauca con 1.222 y Magdalena con 1.209 menores (Palacio, 2016).

\section{Metodología}

Para el desarrollo del presente artículo, se consultaron artículos relacionados con procesos de paz firmados por algunos países como: Guatemala, El Salvador, Perú y México, como también se tomaron algunas referencias para identificar las ventajas y 
desventajas que han tenido estos procesos, relacionándolos con responsabilidad social empresarial e inclusión social, con la finalidad de ser tomados como referencia en el proceso de paz entre Colombia y las Farc, teniendo en cuenta un promedio 18 publicaciones, las cuales se dividen en fuentes primarias y secundarias, donde se toma como referencia artículos en PDF y del mismo modo revistas y periódicos que puedan proporcionar información adicional.

La metodología de la investigación gira en torno a las características de la investigación, ya que utiliza de forma preferente o exclusivamente, información de tipo cualitativo y cuyo análisis se dirige a lograr descripciones detalladas de la investigación objetivo, para lo cual se tendrá en cuenta artículos y documentos relacionados con procesos de paz, con el fin de encontrar aspectos importantes que se puedan aportar al proceso de paz que lleva en país.

\section{Resultados}

Es importante resaltar que durante el tiempo gubernamental del presidente Juan Manuel Santos se identificaron grandes avances en el proceso de paz entre Colombia y las Fuerzas Armadas Revolucionarias de Colombia (Farc), donde se puede resaltar las actitudes que refleja la población civil, presentando un pensamiento diferencial, ya que algunas personas están en contra de los beneficios que se tomaron en cuanta durante los diálogos, siendo los más controversiales:

1. Recibir una renta básica mensual equivalente a 90\% del SMMLV, siempre y cuando no tengan una relación establecida que les genere ingresos.

2. Crean medidas que facilitarían y disminuyan las penas de excombatientes.

Con lo anterior estas alternativas que presenta el gobierno son realizadas con la finalidad de tener en cuenta a la población desmovilizada, para que así vean las oportunidades que pue- den adquirir apoyando la desintegración de este grupo armado Farc, beneficiando principalmente a los campesinos siendo la urbe implicada y afectada directamente por el conflicto. Para fortalecer las expectativas que ofrece el gobierno, se quiere realizar y fundamentar un Centro de Capacitación para Personas Vulnerables enfocado en Desmovilizados (CCPVD), para así disminuir las dificultades que se pueden presentar durante el proceso, de igual forma identificar y dar a conocer la importancia de tener relaciones estrechas con empresas públicas y privadas, con la finalidad de plantear y establecer políticas que beneficien de manera puntual las necesidades y expectativas de esta población al momento de su reintegración en todos los ámbitos.

También se pueden buscar alternativas que faciliten el reintegro, por ende una de la soluciones es que las universidades públicas o privadas puedan ofrecer apoyo y darle a los estudiantes iniciativas de creación de estrategias para ayudar al reintegro de esta población, y del mismo modo proporcionarle opciones de aprendizaje para que así los desmovilizados se puedan capacitar y adquirir conocimiento, con el objetivo de darle dirección a su proyecto de vida y crecimiento personal, esto fundamentado con algunos aspectos que influyeron en el reintegro en los países que se tuvieron en cuenta, y que hoy disfrutan del fortalecimiento y la disminución de problemas sociales, como también realizando centros de encuentro y asociación, esto permitiendo y ayudando a las personas desmovilizadas a contar y a compartir su historia y presentar un acercamiento con más facilidad para su reintegro.

Gracias a la investigación realizada se puede dar un avance significativo en el proyecto que se lleva acabo, tratándose de un Centro de Capacitación para Persona Vulnerables enfocado en Desmovilizados (CCPVD), fortaleciéndolo con lo expuesto anteriormente, y del mismo modo teniendo resultados agradables, ya que al ser presentado en el país de México- Mazatlán, se pudo evidenciar grandes aportes significativos para el avance y del mismo modo gran controversia. 


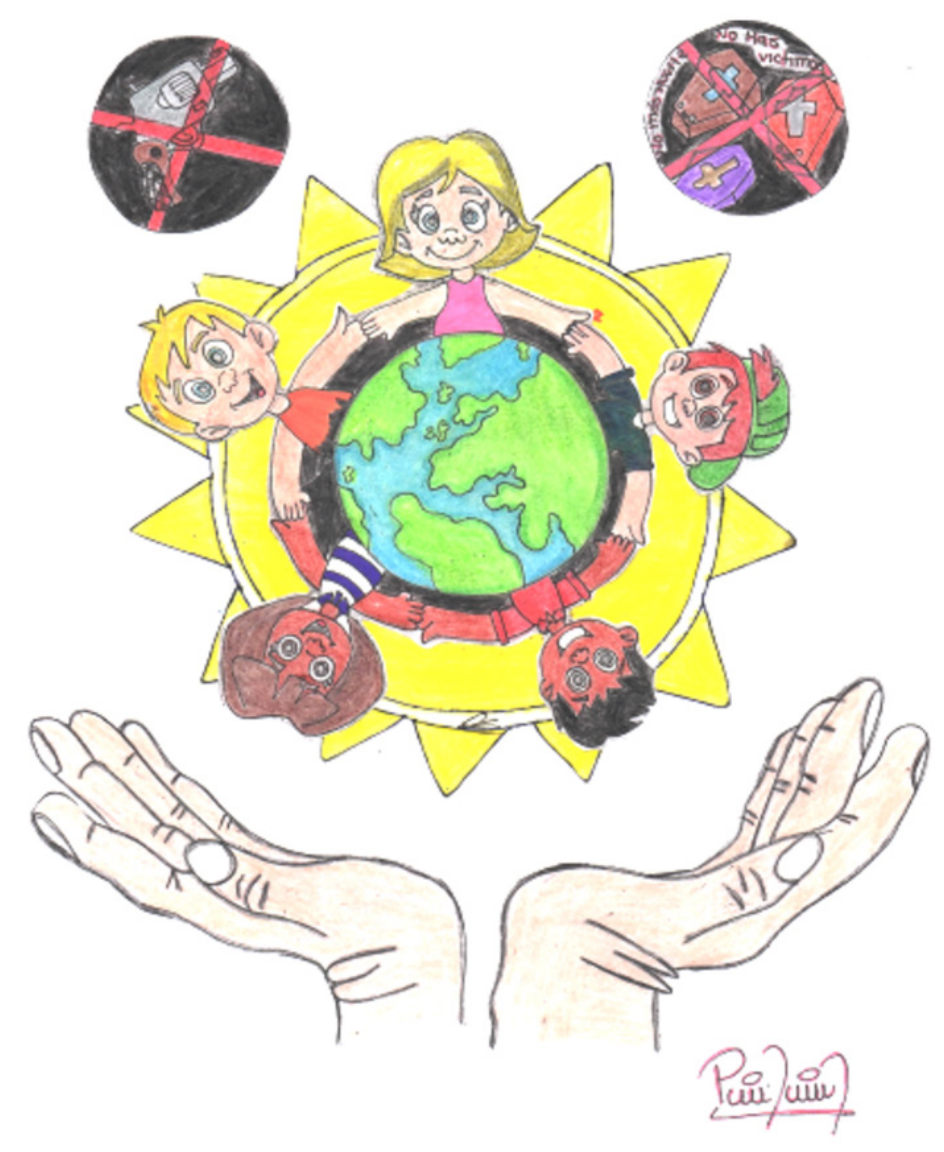

Imagen 1. Todos unidos, podemos hacer más. Fuente. Representación de Paula Andrea León.

La anterior imagen, plasma el sentir de lo que debe ser el significado de paz, una nación donde el respeto a la vida prime, donde los derechos humanos no sean un tema de debate o de cátedra en una universidad, sino que sea una realidad que se aplique para toda la sociedad; que el concepto de perdón sea sincero, no de unos cuantos o de quienes defienden ciertos intereses o colores políticos.

La paz no debe ser un tema del gobierno y sus diferentes entidades, no es un tema de quien escribe sobre la misma, es de todos y cada uno de los ciudadanos, por lo tanto al realizar el proyecto del Centro de Capacitación para Personas Vulnerables enfocado en Desmovilizados (CCPVD), podemos identificar la importancia de estudiantes de administración de empresas u otras carreras profesionales donde existe el compromiso con el proceso de paz, este tema debe ser una prioridad, ya que los niños y jóvenes son los principales implicados, los únicos que van a tener que seguir viviendo en carne viva lo que hasta hoy han tenido que vivir muchos, por lo que cambiar ese pensamiento egoísta va a dejar ver el significado que tiene cada paso que se dé, así sea lo más mínimo, con esto se va identificar lo importante y el impacto tan grande que proporciona, ya que disminuir la violencia no es nada fácil, pero si nos unimos podemos lograr lo que nunca esperábamos, una paz duradera, donde todos pensamos en la estabilidad de todos, primero debemos comenzar en no juzgar, porque todos, absolutamente todos nos hemos equivocado y merecemos una oportunidad, aprender a escuchar es una habilidad que todos tenemos y que no se debe perder, por eso 
no perjudiquemos ni dañemos a nadie con los comentarios que hacemos, por ser diferente o por tener un pasado.

\section{Conclusiones}

En consecuencia, se puede evidenciar que este tema de los grupos de izquierda perjudica el crecimiento económico y aumentan los problemas sociales, por consiguiente la estabilidad económica de la población es baja, también se puede observar que estos grupos realizaban este tipo de organización con el fin de aportar de manera positiva sin tener un impacto negativo. Es importante tener en cuenta el enfoque y la finalidad de los grupos de izquierda, ya que es evidente que el principal objetivo de su creación es ayudar y proporcionar sustento a la población más vulnerable, pero por causa de la falta de apoyo y del conformismo del gobierno, se presentan consecuencias directas en la sociedad. Con lo anterior, los acuerdos de paz es una estrategia muy conveniente para cada país, porque finalmente se aumenta el beneficio de la calidad de vida de cada ciudadano, y disminuye los índices de violencia.

La responsabilidad social empresarial (RSE) es un tema que apunta y se enfoca en el aporte que realizan las empresas para solucionar problemas en cualquier ámbito, ya que la participación de ella aporta de manera directa, al darles oportunidades de ingreso al mundo civil, apoyo económico y del mismo modo un impacto positivo a la calidad de vida de cada persona. También es la principal fuente que puede disminuir la desigualdad e inconformismo hacia la población desmovilizada.

Después de observar e investigar a los países que contaron con la oportunidad de tener esta firma de tratado de paz, se puede resaltar el impacto que trae consigo la firma, y el mismo modo el proceso y las condiciones que deben estar estipuladas en cada uno de ellos, como también brindarles apoyo hasta el punto que sea necesa- rio para evitar fines irreversibles por el incumplimiento.

También se puede decir que las empresas no deberían focalizarse solo en generar utilidades, sino en ver la manera como puede intervenir para solucionar problemas sociales que tal vez no los esté afectando considerablemente en el momento de realizar su actividad económica, pero que, si no se le pone atención y no se buscan estrategias para su solución, puede ser un problema que los obligue a dejar de participar en el mercado.

\section{Referencias}

Alonso, J. (2003). El movimiento zapatista, novedad que rompe las etiquetas. Nómadas, (19), 48-56.

Araoz, S. (2010). Inclusión social: un propósito nacional para Colombia. Bogotá: Universidad Central.

Auger, F. (2013). El movimiento zapatista:¿hacia una verdadera ciudadanía intercultural? Tinkuy: Boletín de Investigación y Debate, (20), 6-22.

Ballesteros, F. (2016). El proceso de paz en el salvador: un referente para Colombia. Maestría en Ciencia Política. Bogotá: Universidad Católica de Colombia. Recuperado de: https://repository. ucatolica.edu.co/bitstream/10983/14198/1/ TESIS\%20PDF\%20CON\%20LICENCIA\%20FREDDY\%20B.\%20.pdf

Blanco, R. (2006). La equidad y la inclusión social: uno de los desafíos de la educación y la escuela hoy. Reice. Revista Iberoamericana sobre Calidad, Eficacia y Cambio en Educación, 4(3), 1-15.

Fedesarrollo (2018). Implementar el acuerdo de paz necesitaría una nueva reforma tributaria: Fedesarrollo. Recuperado de: https:// 
www.fedesarrollo.org.co/content/implementar-el-acuerdo-de-paz-necesitar\%C3\%ADa-una-nueva-reforma-tributaria-fedesarrollo

Fisas, V. (2010). Procesos de paz comparados. ECP. Recuperado de: escolapau.uab.es/img/qcp/ procesos_paz_comparados.pdf

García, V; Aquino, S; Izquierdo, J; Santiago, P. R. (2015). Investigación e innovación en inclusión educativa. Diagnósticos, modelos y propuesta. México: Red Durando de Investigadores Educativos.

Mandoki, L. (Dirección). (2005). Voces inocentes [Película].

Martín, A. (2017). ¿Las Fuerzas Armadas Revolucionarias de Colombia no están desarmadas? Instituto Español de Estudios Estratégicos. Recuperado en: http://www.ieee.es/ Galerias/fichero/docs_informativos/2017/ DIEEEI08-2017_FARC_NoDesarmadas_AGM. pdf

Duque,Y., Cardona, M. \& Rendón, J. (2013). Responsabilidad social empresarial: teorías, índices, estándares y certificaciones. Cuadernos de Administración, 29(50), 196-206.

Padró, E. (2015). Movadef, el pensamiento Gonzalo y la reaparición de Sendero Luminoso: 19922012. Tesis de grado en Ciencia Política y Gobierno. Lima: Pontificia Universidad Católica del Perú. Recuperado en: http://tesis.pucp.edu. pe/repositorio/handle/123456789/7323

Pataquiva, G. N. (2009). Las Farc, su origen y evolución. Unisci Discussion Papers, (19). Recuperado en: http://revistas.ucm.es/index.php/ UNIS/article/view/28644

Reimers, F. (2002). La lucha por la igualdad de oportunidades en América Latina como proceso político. Revista Latinoamericana de Estudios Educativos, XXXII(1), 9-70. Recuperado de: https://www.redalyc.org/pdf/270/27032102.pdf
Semana. (25 de 10 de 2016). El impacto psicosocial de los niños víctimas del conflicto. Recuperado de: https://www.semana.com/educacion/ articulo/impacto-del-conflicto-armado-en-ninos-de-colombia/501110

Swiss Peace (2016). Proceso de paz en: un estudio de caso sobre la negociación y el proceso de diálogo nacional en Guatemala. Swiss Peace / Centro de Estudios de Guatemala (CEG). Recuperado de: https://www.swisspeace.ch/fileadmin/user_ upload/pdf/Mediation/Estudio_de_caso_sobre_Proceso_de_paz_en_Guatemala.pdf

Wabgou, M. \& Quishpe, R. (2014). Participación política de los excombatientes: reflexiones desde Sierra Leona para el caso colombiano. Documentos de Políticas Públicas, (4). Recuperado de: https://www.researchgate.net/ publication/320149979_PARTICIPACION_POLITICA_DE_LOS_EXCOMBATIENTES_REFLEXIONES_DESDE_SIERRA_LEONA_ PARA_EL_CASO_COLOMBIANO 